\section{Reduced risk of ICD shock with amiodarone plus $\beta$-blocker}

Shocks from implantable cardioverterdefibrillators (ICDs) often occur in the first year after implantation. These events result from recurrent ventricular tachycardia and fibrillation, as well as-perhaps unexpectedly-from atrial arrhythmias. These high-voltage shocks are painful and can lead to premature depletion of the ICD battery. Although $\beta$-blockers (metoprolol, carvedilol or bisoprolol) are standard therapy for most patients with ICDs, investigators for the Optimal Pharmacological Therapy in Cardioverter Defibrillator Patients (OPTIC) trial sought to find out whether other antiarrhythmic therapies were effective in preventing ICD shocks.

A total of 412 patients who had received a dual-chamber ICD for sustained or inducible ventricular tachycardia or fibrillation were recruited to the trial. Patients were randomized to receive standard $\beta$-blocker therapy, amiodarone plus a $\beta$-blocker, or sotalol alone. Treatment efficacy was compared in terms of the first ICD shock over a median follow-up of 359 days.

Risk of an ICD shock decreased by about fourfold in patients receiving amiodarone plus $\beta$-blocker compared with those receiving a $\beta$-blocker alone $(P<0.001)$. ICD shocks also occurred less frequently among patients receiving sotalol than among those receiving only a $\beta$-blocker, but the results were not statistically significant. Drug-related adverse effects, including pulmonary and thyroid events and symptomatic bradycardia, were more common among patients assigned to amiodarone than among those assigned to standard $\beta$-blocker therapy. The authors conclude that the potential benefits of amiodarone therapy, in terms of improved quality of life, might outweigh the risk of side effects.

Claire Braybrook

Original article Connolly SJ et al. (2006) Comparison of $\beta$-blockers, amiodarone plus $\beta$-blockers, or sotalol for prevention of shocks from implantable cardioverter defibrillators: the OPTIC study: a randomized trial. JAMA 295: 165-171

\section{Fat intake and risk of cardiovascular disease: the WHI dietary modification trial}

The Women's Health Initiative dietary modification trial investigated whether a low-fat, high-fiber (from vegetables, fruits and grains) diet could reduce the incidence of breast and colorectal cancer in postmenopausal women; the effect on cardiovascular disease was a secondary assessment.

Altogether, 48,835 women aged 50-79 years, of diverse backgrounds and ethnicities, entered the 8-year study. Participants were randomized to follow their normal diet but be given dietary education materials $(n=29,294)$, or to follow a program designed to lower fat intake to $20 \%$ of total calorie intake and to increase average daily fruit and vegetable consumption from 3.6 to $\geq 5.0$ servings, and grain consumption from 4.7 to $\geq 6.0$ servings $(n=19,541)$. Diets were monitored by self-completed questionnaires.

Average fat consumption on the modified diet was $23.8 \%$ of total calorie intake after 1 year and $28.8 \%$ after 6 years. Consumption of fruit and vegetables increased by about one serving, and grains by half a serving daily. The annual incidences of coronary heart disease, stroke or cardiovascular disease did not decrease significantly compared with the normal diet group. A trend towards reduced risk of coronary heart disease was found only among women who consumed substantially less saturated and trans fat or notably more vegetables and fruits.

The lack of focus on specific fats and nutrients with established links to cardiovascular disease, potential bias through self-reporting, and lack of data on how such a diet affects men or younger people limit this study's public health impact.

Claire Braybrook

Original article Howard LBV et al. (2006) Low-fat dietary pattern and risk of cardiovascular disease: the Women's Health Initiative Randomized Controlled Dietary Modification Trial. JAMA 295: 655-666

\section{C-reactive protein is a poor predictor of subclinical atherosclerosis}

Elevated levels of C-reactive protein (CRP) are associated with a raised risk of incident cardiovascular events. How CRP mediates these effects is unclear, but high concentrations of this biomarker have been proposed to indicate an increased burden of atherosclerosis. Studies investigating the relationship between CRP and atherosclerotic burden have yielded conflicting findings or have used insensitive 